\title{
Incidence and Predictors of Complications of Acute Achilles Tendon Rupture Repair at Hamad General Hospital, Doha, Qatar
}

\author{
Sameh M. Abolfotouh ${ }^{1}$, Mohamed A. Al Dosari ${ }^{1}$, Nader Sayed ${ }^{1}$, \\ Hussam Banna ${ }^{1}$, Mostafa A. Abolfotouh ${ }^{2}$ \\ ${ }^{1}$ Hamad General Hospital, Doha, Qatar \\ ${ }^{2}$ King Abdullah International Medical Research Center, Riyadh, KSA \\ Email: mabolfotouh@gmail.com
}

Received December 15, 2013; revised January 10, 2014; accepted January 18, 2014

Copyright (c) 2014 Sameh M. Abolfotouh et al. This is an open access article distributed under the Creative Commons Attribution License, which permits unrestricted use, distribution, and reproduction in any medium, provided the original work is properly cited. In accordance of the Creative Commons Attribution License all Copyrights (C) 2014 are reserved for SCIRP and the owner of the intellectual property Sameh M. Abolfotouh et al. All Copyright (C) 2014 are guarded by law and by SCIRP as a guardian.

\section{ABSTRACT}

Aim: The aims of this study were: 1) to estimate the prevalence and pattern of complications after Achilles tendon (AT) repair, and 2) to determine the significant predictors of post-operative infection. Methods: A retrospective cohort study of all patients who were operated at Hamad General Hospital (HGH) between June 2010 and June 2012 for AT rupture $(n=102)$, was conducted. Data was collected on 1) patient' characteristics such as age, sex; 2) disease characteristics such as mechanism of rupture, type of rupture (partial or complete), whether an anterior or posterior slab was applied, number of suture materials, number of antibiotics, surgical time, time to surgery and length of hospital stay (LOS), number of follow up visits, and 3) complications. Descriptive and analytical statistical analyses were applied. Receiver operating characteristic curve was applied to identify the validity of different LOS values, with a significance level at $p \leq \mathbf{0 . 0 5}$. Results: Of the 102 patients with Achilles rupture, almost males $(96.1 \%)$, with a mean age $31.07 \pm 9.71$ years, $52 \%$ with complete rupture, the majority were open ruptures $(81.4 \%)$ and bathroom-related $(\mathbf{7 0 . 6} \%)$. Anterior slab was applied to $58.2 \%$ and 2 types of suture materials to $71.6 \%$ of cases. Fifteen cases $(14.7 \%$, 95\% CI: $7.8 \%-21.6 \%)$ presented with one or more complications (9.8\% post-operative infections, $5.9 \%$ stiffness and $2 \%$ re-rupture). Post-operative infections were significantly associated with: old age $(z=2.11, p=0.035)$, longer $\operatorname{LOS}(z=2.01, p=0.04)$, and presence of diabetes (Fisher exact test: $p=0.003)$. After adjustment for age, LOS $(p=0.04)$ and diabetes $(p=0.017)$ remained as significant predictors of post-operative infections. LOS of 2.5 days was the optimum cut-off point above which post-operative infection is more likely to occur, with sensitivity of $80 \%$ and specificity of $54 \%$. Conclusion: Achilles repair post operative infection ranks first as a complication of AT surgical repair, and its incidence is relatively higher in HGH than the counterpart figures in the literature. The presences of diabetes and LOS are independent predictors of this infection. Further prospective studies are recommended to control for all possible confounders of outcome of AT rupture repair.

\section{KEYWORDS}

Achilles Tendon; Repair; Surgical; Outcome; HGH; Qatar

\section{Introduction}

The Achilles Tendon (AT) is one of the most frequently ruptured tendons in the human body [1] and has become the third most frequent major tendon injury behind those of the rotator cuff and knee-extensor mechanisms [2]. There has been an increase in incidence over the last half century [3], with studies reporting up to 18 per 100,000 [1]. Acute ruptures of the Achilles tendon most commonly affect individuals in their third to fifth decade of life who are intermittently active [4]. The etiology of Acute Achilles tendon ruptures is multi-factorial and includes overuse injuries, host factors, medications, or inappro- 
priate footwear [5].

Recent studies have shown successful outcomes with non-operative and operative treatment. Comparative studies between non-operative and operative treatment showed a higher rate of re-rupture with nonoperative treatment, but also showed risks of wound healing, wound infection, and nerve injury with operative treatment [4-8]. Studies have published a re-rupture rate of $4.3 \%$ to $23 \%$ in non-operatively treated patients compared to $1.7 \%$ to $10 \%$ for operatively treated patients [9]. Operative treatment was associated with a significantly higher risk of wound complications, adhesions, infection, and nerve injury [10].

A large number of medical reports and meta-analyses have been published in the field of Achilles tendon rupture, but there is still a lack of consensus on the best management. There is limited knowledge about the predictive factors for outcome after an acute Achilles tendon rupture. The identification of important predictive factors would be beneficial for both an understanding of how to individualize treatments and when designing future treatment protocols [11]. The aims of this study were: 1 ) to estimate the incidence and pattern of complications after Achilles tendon repair, and 2) to determine the significant predictors of post-operative infection.

\section{Methodology}

\subsection{Setting}

This study was conducted at Hamad General Hospital (HGH), at Doha, Qatar. It is the only primary trauma center in the state of Qatar. It's the 2nd hospital in the world to be accredited by the Accreditation Council for Graduate Medical Education International (ACGMEI). HGH is the first and only hospital corporation in the world to achieve simultaneous accreditation and reaccreditation of all its facilities by the Joint Commission International (JCI), and Qatar is the only country outside of the United States to achieve such accreditation for all its public hospitals. It has a capacity of 603 beds and one of the highest rates of trauma in the Middle East [12,13].

\subsection{Design}

This observational retrospective cohort study involved the review of hospital charts for patients receiving Achilles tendon repair surgery over a 2-year period (June 2010-June 2013, inclusive) at Hamad General Hospital. The existing practice pattern for Achilles tendon surgery at HGH is that patients are admitted for several hours up to a few days before an operating room becomes available. However, for open injuries, surgery is performed as an emergency within 24 hours, although an 8-hour interval is a recommendation. After surgery, patients are either discharged the same day or thereafter, depending on their postoperative status. Currently, there is no agreedupon standard of care for the pre- and/or postoperative management of Achilles tendon ruptures in HGH.

\subsection{Data Collection and Analysis}

Following the approval of the study protocol by the Institutional Review Board (IRB) committee of Hamad General Hospital, the following data were collected.

- Patient characteristics such as age, sex, co-morbidities, etc.

- Disease characteristics such as type of injury (closed or open), mechanism of rupture (sport-related or others), type of rupture (partial or complete).

- Management characteristics: number of suture materials, number of antibiotics, surgical time and length of hospital stay (LOS), number of follow up visits, and complications. These complications are infection (superficial or deep), stiffness and re-rupture problems.

All data were coded and analyzed using the Statistical Package for the Social Sciences (SPSS) program (version 11). Descriptive statistics such as mean, median, range, and standard deviation were used. Frequencies (\%) with their corresponding 95\% confidence intervals (CIs) were calculated. Both qualitative and quantitative analyses were applied to investigate associations and differences. Student $t$ test and Mann-Whitney $U$ test were used to compare numerical data. For categorical data, chi-square test and Fisher exact test were applied. Logistic regression analyses were applied to identify the significant predictors of AT repair post-operative infection. Receiver operating characteristic (ROC) curve was applied to allocate the cut-off value of LOS above which infection is likely to occur post-operatively. Significance was considered at $p \leq 0.05$.

\section{Results}

\subsection{Patients, Injury and Management Characteristics}

Table 1 shows the distribution of the study sample according to incidence of infection and association with some other characteristics. Cases were mostly of male gender $96 \%$, aging from 10 to 68 years, with the mean age of $31.1 \pm 9.7$ years, with partial and complete ruptures of $48 \%$ and $52 \%$ respectively, mostly due to bathroom injury $70 \%$, with anterior (58\%) and posterior (42\%) slabs applied.

\subsection{Incidence of Complications}

Complications occurred in 14.7\% (95\% CI: 7.8 - 21.6) of all cases. These complications were presented as; infection 9.8\% (95\% CI: 4.0 - 15.6), stiffness 5.9\% (95\% 
Table 1. Association of infection after Achilles tendon rupture repair with some patients, injury and management characteristics.

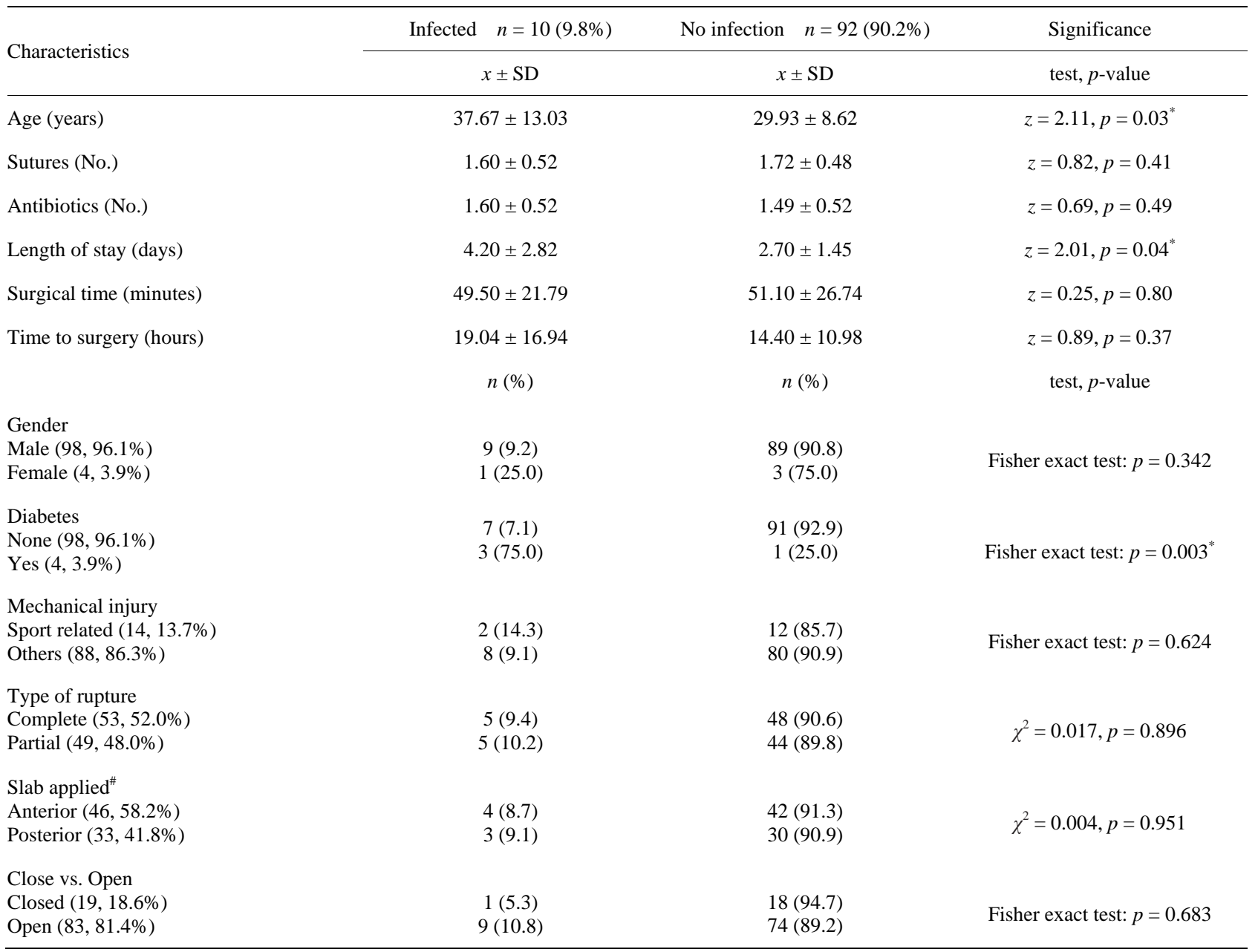

z: Mann-Whitney test; $\chi^{2}$ : Pearson Chi-square; ${ }^{*}$ Statistical significance; ${ }^{\#}$ Figures were shown for available data.

CI: 1.3 - 10.5) and re-rupture 2\% (95\%CI: -0.7 - 4.7), (Figure 1).

\subsection{Association of Infection with Some Patients, Injury and Management Characteristics}

Incidence of infection was not significantly associated with any of the injury characteristics: mechanism of rupture, its type, complete or partial, or close or open. The presence of diabetes was the only variable significantly associated with infection, with incidence of $75 \%$ among diabetics as compared to only $7.1 \%$ among non-diabetics. There was a tendency for higher-though not statistically significant-incidence of infection among females (25\% vs. 9.2\%). Meanwhile, of all management characteristics, length of hospital stay was the only variable significantly associated with infection. Infected cases showed significantly higher mean length of stay (4.2 days) than did the non-infected ones (2.7 days).

When we applied the regression analysis of post-repair infection with both length of stay and the presence of di-

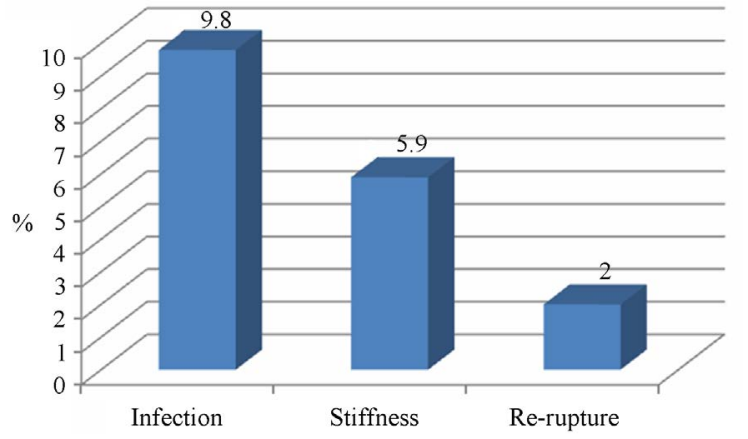

Figure 1. Incidence of complications after Achilis tendon rupture repair at $\mathrm{HGH}$.

abetes as independent variables (these two variables were the only variables significantly associated with infection in the univariate analyses), and adjusting for age, the presence of diabetes was the only significant predictor of post-repair infection (Table 2). Diabetics are 30 times more likely to contract infection after repair than nondiabetics $(\mathrm{OR}=30.58)$. 
Table 2. Age adjusted predictors of post-operative infection.

\begin{tabular}{ccccc}
\hline & Beta & S.E. & adjp-value & adjOR (95\% CI) \\
\hline Gender (Male vs. Female) & -0.40 & 1.68 & 0.81 & $0.67(0.03-17.99)$ \\
Hospital stay (Days) & 0.37 & 0.18 & $0.04^{*}$ & $1.45(1.02-2.08)$ \\
Diabetes (Yes vs. No) & 3.421 & 1.429 & $0.017^{*}$ & $30.58(1.85-503.41)$ \\
Type of injury (Open vs. Closed) & 1.09 & 0.86 & 0.20 & $2.97(0.55-16.00)$ \\
Mechanism (Bathroom vs. Sport) & 0.67 & 0.88 & 0.45 & $1.95(0.35-10.89)$ \\
Type of rupture (Complete vs. Partial) & 0.19 & 0.60 & 0.74 & $1.21(0.38-3.90)$ \\
Slab application (Posterior vs. Anterior) & 0.02 & 0.75 & 0.98 & $1.02(0.24-4.31)$ \\
Antibiotics (No.) & 0.41 & 0.57 & 0.47 & $1.51(0.50-4.58)$ \\
Suture materials (No.) & 0.37 & 0.67 & 0.58 & $1.45(0.39-5.41)$ \\
Surgical time (minutes) & -0.01 & 0.01 & 0.63 & $0.99(0.97-1.02)$ \\
Time to surgery (hr) & -0.001 & 0.02 & 0.97 & $0.99(0.95-1.05)$ \\
\hline
\end{tabular}

*Denotes significance; OR: odds ratio.

When applying the Receiver operating characteristic (ROC) curve to allocate the cut-off of hospital days above which infection is more likely to occur, the value of 2 and half days was the cut-off for post-repair infection. At this cut-off of 2.5 days, sensitivity was $80 \%$ (high), while the specificity was only $54 \%$ (low).

However, the negative predictive value was very high (96\%). This means that when a patient is discharged before the period of 2.5 days, we will be more confident of no post-repair infection contraction (Table 3 and Figure 2).

\section{Discussion}

The etiology of Achilles tendon ruptures is regarded as multifactorial [13], but there is little agreement in the literature Achilles tendon ruptures commonly occur to otherwise healthy men in the middle age group who have had no previous injury or problem reported in the affected leg. In a previous study by Houshian et al. [14], the incidence of rupture was highest in the 30 - 39 year age group. This was in agreement with the finding of the present study, were the mean age of patients with ruptures was 31.1 years, and infection was even significantly associated with increasing age. There is some evidence of degenerative changes in the ruptured tendon [13,15, 16], and these changes, combined with a high activity level, may partly explain the sports-related peak in incidence in the middle-aged group.

Most Achilles tendon ruptures occur in men and the ratio between men and women is between 3:1 and 18:1, in general approximately 10:1 [14,17]. This was in agreement with the findings of the present study, where almost all patients who presented to the hospital with Achilles
Table 3. $2 \times 2$ table of length of stay and post-operative infection after Achillis tendon rupture repair.

\begin{tabular}{cccc}
\hline & Infection & No infection & Total \\
\hline 2.5 Days or more & $8(20.0)$ & $42(45.7)$ & 50 \\
Less than 2.5 days & $2(20.0)$ & $50(54.3)$ & 52 \\
Total & 10 & 92 & 102 \\
\hline
\end{tabular}

Sensitivity $=8 / 10=80 \%$, Specificity $=50 / 92=54.3 \%$, Positive predictive value $(\mathrm{PPV})=8 / 58=14 \%$, Negative predictive value $(\mathrm{NPP})=50 / 52=$ $96.2 \%$.

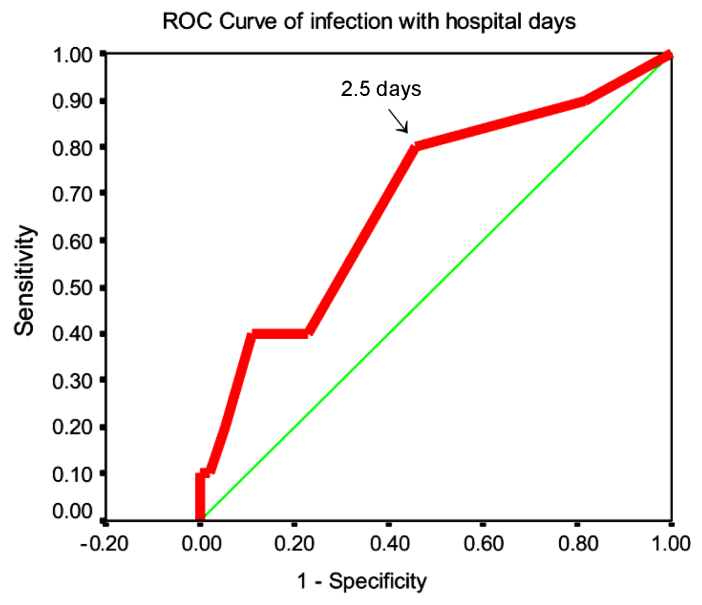

Diagonal segments are produced by ties

Figure 2. ROC curve of hospital stay in days with complications.

tendon rupture (96\%) were of male gender. The incidence of Achilles tendon rupture appears to be rising and approximately $75 \%$ of all ruptures occur during sporting activities [13]. However, in the present study, sports-re- 
lated ruptures constituted only $14 \%$ of all ruptures, while the majority of ruptures were due to bathroom injury. This may reflect the necessity to look for an ergonomicbased design to the bathrooms in our Gulf region that reduces the incidence of such type of injury.

The treatment of an Achilles tendon rupture is either surgical or nonsurgical. However, there is a lack of literature indicating which treatment leads to superior functional outcomes [18-22]. Studies have published a rerupture rate of $4.3 \%$ to $23 \%$ in non-operatively treated patients compared to $1.7 \%$ to $10 \%$ for operatively treated patients [11]. In the present study, those who presented with re-rupture constituted only $2 \%$ of all surgical cases. This figure is less than the figure from four previous studies $[8,18,20,23]$ of open surgical treatment versus nonsurgical treatment that showed a re-rupture rate of $3.5 \%$ in the surgically treated group and $12.6 \%$ in the nonsurgically-treated group. Studies of mini-invasive techniques indicate a decreased incidence of wound complications, especially infection, but this technique might increase the risk of sural nerve injury [24].

Operative treatment was associated with a significantly higher risk of wound complications, adhesions, infection, and nerve injury [10]. An incidence of $4.7 \%$ postoperative infection was reported from four previous studies [18-20,23], supported by a large meta-analysis study [25], suggesting that there are benefits to non-operative management. In the present study, post-operative infection showed a comparatively higher incidence of $10 \%$, supporting the results of previous studies concerning complications other than re-rupture. Surgical treatment may be able effectively to reduce the risk of reruptures but leads to more complications than non-surgical treatment. The re-rupture rate is relatively low regardless of treatment and might therefore not be the most appropriate outcome measurement when comparing treatments.

There is limited knowledge about the predictive factors for outcome after an acute Achilles tendon rupture. The identification of important predictive factors would be beneficial for both an understanding of how to individualize treatments and when designing future treatment protocols [10]. In a previous study, age was the only independent predictor of outcome, while gender, body weight, height, period between rupture and operation, surgeon, rupture site, operative method, complications, and thickness, width, and area of the Achilles tendon at follow up were all not related significantly to the outcome [26]. In the present study, age, length of stay, as well as the presence of diabetes were significantly associated with infection, and when adjusting for age as a possible confounder, the presence of diabetes and longer length of stay remained as significant predictors of postoperative infection.
A higher rate of deep infection has been reported in diabetic patients undergoing surgery [27]. In patients with operatively managed Achilles tendon ruptures, diabetes increased the risk of wound complications by 3.4 times [28]. A deep infection after surgery for AT repair can have devastating effects, and is a major management challenge. In the present study, the presence of diabetes was a significant predictor of post-operative infection, even after adjusting for age. Infection was shown in $75 \%$ of diabetic patients as compared to only $7.1 \%$ in nondiabetic ones. However, the finding that diabetic patients have high rate of post-operative infection is not new [27, 28], and it is also of note the fact that tendon healing in these patients impaired [29]. Percutaneous repair in diabetic patients with AT rupture may minimize the rate of infections arising from traditional open repairs [30-32].

Surgical management is currently the most common treatment offered for ruptured Achilles tendon; however, the length of hospital stay varies among hospitals. In the present study, the length of stay ranged from 1 to 11 days with an average stay of 2.8 days. Longer LOS was significantly associated with post-operative infection. An observational retrospective analytical study of safety and hospital stay cost of AT surgery demonstrated that surgical treatment of acute Achilles tendon ruptures was both safe and less costly when performed as an outpatient procedure [31].

The infection rate reported in the literature is consistent with that of this study [22]. When applying ROC curve to allocate the cut-off at which infection is predicted, a LOS of 2.5 days or less would be protective against post-operative infection. When applying the ROC curve to allocate the cut-off of hospital days above which infection is more likely to occur, the value of 2 and half days was the cut-off for post-repair infection. At this cutoff of 2.5 days, sensitivity was $80 \%$ (high), while the specificity was only $54 \%$ (low). However, the negative predictive value was very high (96\%). This means that when a patient is discharged before the period of 2.5 days, we will be almost sure of no infection contraction. That is a good negative test. Minimally invasive surgical (MIS) repair using Achilles method can achieve smaller incisions, shorter operative time and hospital stay [22].

\section{Limitations}

The nature of this observational analytical study limits our ability to capture reasons for prolonged admission times. The inconsistent details recorded in the hospital charts did not allow for an analysis of co-morbidities, other than diabetes mellitus. Therefore, the effects of smoking status, concurrent medication or activity level on prolonged admission times could not be determined in this study. The main outcome of the present study has been complications such as re-ruptures and infections, 
without focusing on the patient-reported or functional outcomes relevant to the majority of patients who do not experience these complications.

\section{Conclusions}

Aside from the above mentioned limitations, we can conclude the followings: Incidence of post-operative complications is seemingly high when compared with the international figures. Infection ranked first as a post-repair complication, followed by stiffness and re-repair. Early discharge of post-repair for Achilles is recommended as a safeguard against infection.

Special attention must be paid to Achilles cases with diabetes. Percutaneous repair of the AT is a viable option for diabetic patients. Future studies should prospectively analyze patients and stratify them according to risk factors that could potentially require admission or delay discharge after a surgical procedure. Finally, major technical improvements in surgical and non-surgical treatment may change the advantages and disadvantages of each treatment.

\section{Acknowledgements}

This study has been approved by the IRB of Hamad General Hospital, Doha, Qatar. Authors would like to thank Mr. Mahmoud Salam for his efforts in designing the graphical and tabular presentations of the manuscript.

\section{REFERENCES}

[1] J. Leppilahti, J. Puranen and S. Orava, "Incidence of Achilles Tendon Rupture,” Acta Orthopaedica Scandinavica, Vol. 67, No. 3, 1996, pp. 277-279. http://dx.doi.org/10.3109/17453679608994688

[2] A. Lesic and M. Bumbasirevic, "Disorders of the Achilles Tendon,” Journal of Orthopaedic Trauma, Vol. 18, 2004, pp. 63-75.

[3] J. Ufberg, R. A. Harrigan, T. Cruz, et al., "Orthopedic Pitfalls in the ED: Achilles Tendon Rupture," American Journal of Emergency Medicine, Vol. 22, No. 7, 2004, pp. 596-600. http://dx.doi.org/10.1016/j.ajem.2004.09.007

[4] J. M. Weatherall, K. Mroczek and N. Tejwani, "Acute Achilles Tendon Ruptures,” Orthopedics, Vol. 33, No. 10, 2010, pp. 758-764.

http://dx.doi.org/10.3928/01477447-20100826-21

[5] M. Kvist, “Achilles Tendon Injuries in Athletes,” Annales Chirurgiae et Gynaecologiae, Vol. 80, No. 2, 1991, pp. 188-201.

[6] T. M. Hufner, D. B. Brandes, H. Thermann, M. Richter, K. Knobloch and C. Krettek, "Long-Term Results after Functional Nonoperative Treatment of Achilles Tendon Rupture,” Foot \& Ankle International, Vol. 27, No. 3, 2006, pp. 167-171.

[7] M. Möller, T. Movin, H. Granhed, K. Lind, E. Faxén and
J. Karlsson, “Acute Rupture of Tendon Achillis. A Prospective Randomised Study of Comparison between Surgical and Non-Surgical Treatment," The Journal of Bone \& Joint Surgery, Vol. 83, No. 6, 2001, pp. 843-848. http://dx.doi.org/10.1302/0301-620X.83B6.11676

[8] M. L. Costa, K. MacMillan, D. Halliday, et al., "Randomised Controlled Trials of Immediate Weight-Bearing Mobilization for Rupture of the Tendo Achilles," The Journal of Bone \& Joint Surgery, Vol. 88, No. 1, 2006, pp. 69-77.

http://dx.doi.org/10.1302/0301-620X.88B1.16549

[9] B. C. Twaddle and P. Poon, "Early Motion for Achilles Tendon Ruptures: Is Surgery Important? A Randomized, Prospective Study," The American Journal of Sports Medicine, Vol. 35, No. 12, 2007, pp. 2033-2038. http://dx.doi.org/10.1177/0363546507307503

[10] R. J. Khan, D. Fick, A. Keogh, J. Crawford, T. Brammar and M. Parker, "Treatment of Acute Achilles Tendon Ruptures. A Meta-Analysis of Randomized, Controlled Trials," The Journal of Bone \& Joint Surgery, Vol. 87, No. 10, 2005, pp. 2202-2210.

http://dx.doi.org/10.2106/JBJS.D.03049

[11] N. Olsson, "Acute Achillis Tendon Rupture. Outcome, Prediction and Optimized Treatment," University of Gthenburg, Gothenburg, Department of Orthopedics, Institute of Clinical Science, 2013.

[12] Hamad Medical Corporation, 2013. http://www.hmc.org.qa/en/

[13] S. W. Waterston, N. Maffulli and S. W. Ewen, "Subcutaneous Rupture of the Achilles Tendon: Basic Science and Some Aspects of Clinical Practice," British Journal of Sports Medicine, Vol. 31, No. 4, 1997, pp. 285-298. http://dx.doi.org/10.1136/bjsm.31.4.285

[14] S. Houshian, T. Tscherning and P. Riegels-Nielsen, "The Epidemiology of Achilles Tendon Rupture in a Danish county,” Injury, Vol. 29, No. 9, 1998, pp. 651-654. http://dx.doi.org/10.1016/S0020-1383(98)00147-8

[15] O. Arner, A. Lindholm and S. R. Orell, "Histologic Changes in Subcutaneous Rupture of the Achilles Tendon; a Study of 74 Cases," Acta Chirurgica Scandinavica, Vol. 116, No. 5-6, 1959, pp. 484-490.

[16] N. Maffulli, U. G. Longo, G. D. Maffulli, C. Rabitti, A. Khanna and V. Denaro, "Marked Pathological Changes Proximal and Distal to the Site of Rupture in Acute Achilles Tendon Ruptures,” Knee Surgery, Sports Traumatology, Arthroscopy, Vol. 19, No. 4, 2011, pp. 680-687. http://dx.doi.org/10.1007/s00167-010-1193-2

[17] A. Cretnik and A. Frank, "Incidence and Outcome of Rupture of the Achilles Tendon," Wiener Klinische Wochenschrift, Vol. 116, No. 2, 2004, pp. 33-38.

[18] R. Cetti, S. E. Christensen, R. Ejsted, et al., “Operative versus Nonoperative Treatment of Achilles Tendon Rupture. A Prospective Randomized Study and Review of the Literature," The American Journal of Sports Medicine, Vol. 21, No. 6, 1993, pp. 791-799. http://dx.doi.org/10.1177/036354659302100606

[19] A. E. Inglis, W. N. Scott, T. P. Sculco, et al., "Ruptures of the Tendo Achillis. An Objective Assessment of Sur- 
gical and Non-Surgical Treatment," The Journal of Bone \& Joint Surgery, Vol. 58, No. 7, 1976, pp. 990-993.

[20] L. Nistor, "Surgical and Non-Surgical Treatment of Achilles Tendon Rupture. A Prospective Randomized Study," The Journal of Bone \& Joint Surgery, Vol. 63, No. 3, 1981, pp. 394-399.

[21] L. J. Lapidus, S. Rosfors, S. Ponzer, et al., "Prolonged Thromboprophylaxis with Dalteparin after Surgical Treatment of Achilles Tendon Rupture: A Randomized, Placebo-Controlled Study," Journal of Orthopaedic Trauma, Vol. 21, No. 1, 2007, pp. 52-57. http://dx.doi.org/10.1097/01.bot.0000250741.65003.14

[22] M. Bhandari, G. H. Guyatt, F. Siddiqui, et al., "Treatment of Acute Achilles Tendon Ruptures: A Systematic Overview and Metaanalysis," Clinical Orthopaedics and Related Research, Vol. 400, 2002, pp. 190-200. http://dx.doi.org/10.1097/00003086-200207000-00024

[23] L. M. Schroeder and K. Steinbrueck, “Treatment of Acute Achilles Tendon Ruptures: Open vs. Percutaneous Repair vs. Conservative treatment. A Prospective Randomized Study," Orthopaedic Transactions, Vol. 21, No. 4, 1997, p. 1228.

[24] R. Metz, G. J. Van der Heijden, E. Verleisdonk, N. Kolfschoten, M. H. Verhofstad and C. Van der Werken, "Effect of Complications after Minimally Invasive Surgical Repair of Acute Achilles Tendon Ruptures: Report on 211 Cases," The American Journal of Sports Medicine, Vol. 39, No. 4, 2011, pp. 820-824. http://dx.doi.org/10.1177/0363546510392012

[25] M. P. Jones, R. J. Khan and R. L. Carey Smith, "Surgical Interventions for Treating Acute Achilles Tendon Rupture: Key Findings from a Recent Cochrane Review," The Journal of Bone \& Joint Surgery, Vol. 94, No. 12, 2012, p. 88.

[26] J. Leppilahti, K. Forsman, J. Puranen and S. Orava, “Outcome and Prognostic Factors of Achilles Rupture Repair
Using a New Scoring Method," Clinical Orthopaedics and Related Research, Vol. 346, 1998, pp. 152-161. http://dx.doi.org/10.1097/00003086-199801000-00022

[27] J. Aderinto and J. F. Keating, "Intramedullary Nailing of Fractures of the Tibia in Diabetics," Journal of Bone and Joint Surgery, Vol. 90, No. 5, 2008, pp. 638-642. http://dx.doi.org/10.1302/0301-620X.90B5.19854

[28] N. Bruggeman, N. S. Turner, D. L. Dahm, A. E. Voll, T. L. Hoskin, D. J. Jacofsky and G. J. Haidukewych, "Wound Complications after Open Achilles Tendon Repair: An Analysis of Risk Factors," Clinical Orthopaedics and Related Research, Vol. 427, 2004, pp. 63-66. http://dx.doi.org/10.1097/01.blo.0000144475.05543.e7

[29] J. Nestorson, T Movin, M. Moller and J. Karlsson, "Function after Achilles Tendon Rupture in the Elderly: 25 Patients Older than 65 Years Followed for 3 Years," Acta Orthopaedica Scandinavica, Vol. 71, No. 1, 2000, pp. 64-68. http://dx.doi.org/10.1080/00016470052943928

[30] N. Maffulli, U. G. Longo, G. D. Maffulli, A. Khanna and V. Denaro, "Achilles Tendon Ruptures in Diabetic Patients," Archives of Orthopaedic and Trauma Surgery, Vol. 131, No. 1, 2011, pp. 33-38. http://dx.doi.org/10.1007/s00402-010-1097-0

[31] D. P. Goel, D. Chan, K. Watson and N. Mohtadi, "Can Platelet-Rich Plasma Enhance Tendon Repair?” Canadian Journal of Surgery, Vol. 6, 2009, pp. 467-472.

[32] A. P. Chan, Y. Y. Chan, D. T. Fong, P. Y. Wong, H. Y. Lam, C. K. Lo, P. S. Yung, K. Y. Fung and K. M. Chan, "Clinical and Biomechanical Outcome of Minimal Invasive and Open Repair of the Achilles Tendon," Sports Medicine, Arthroscopy, Rehabilitation, Therapy \& Technology, Vol. 3, No. 1, 2011, p. 32.

http://dx.doi.org/10.1186/1758-2555-3-32 